Research Paper

\title{
Salmonella reduces tumor metastasis by downregulation C-X-C chemokine receptor type 4
}

\author{
Tai-Huang Lee1, Gaun-You Lin², Ming-Hui Yang33, Yu-Chang Tyan4, Che-Hsin Lee2,5,6,7凶 \\ 1. Department of Internal Medicine, Kaohsiung Municipal Ta-Tung Hospital, Kaohsiung Medical University Hospital, Kaohsiung Medical University, \\ Taiwan. \\ 2. Department of Biological Sciences, National Sun Yat-sen University, Kaohsiung, Taiwan. \\ 3. Department of Medical Education and Research, Kaohsiung Veterans General Hospital, Kaohsiung, Taiwan \\ 4. Department of Medical Imaging and Radiological Sciences, Kaohsiung Medical University, Kaohsiung, Taiwan. \\ 5. Department of Medical Research, China Medical University Hospital, China Medical University, Taichung, Taiwan. \\ 6. Department of Medical Laboratory Science and Biotechnology, Kaohsiung Medical University, Kaohsiung, Taiwan. \\ 7. International Ph.D. Program for Science, National Sun Yat-sen University, Kaohsiung, Taiwan.
}

$\square$ Corresponding author: Dr. Che-Hsin Lee, Department of Biological Sciences, National Sun Yat-sen University, Kaohsiung, Taiwan, 70 Lienhai Rd. Kaohsiung 80424, Taiwan. E-mail: chlee@mail.nsysu.edu.tw.

(1) The author(s). This is an open access article distributed under the terms of the Creative Commons Attribution License (https://creativecommons.org/licenses/by/4.0/). See http://ivyspring.com/terms for full terms and conditions.

Received: 2021.03.15; Accepted: 2021.05.19; Published: 2021.06.01

\begin{abstract}
Tumor metastasis is the main reason for the death of most cancer patients. C-X-C chemokine receptor type 4 (CXCR4) has been demonstrated to be overexpressed in numerous types of cancer. CXCR4 selectively binds with stromal cell-derived factor 1 (SDF1), also known as C-X-C family chemokine ligand 12 (CXCL12) (CXCL12/SDF-1), which induced tumor proliferation and metastasis. Recently, the use of conventional cancer treatments had some limitation; bacteria treatment for cancer becomes a trend that overcomes these limitations. Plenty of studies show that Salmonella has anti-tumor and anti-metastatic activity. The current study aimed to investigate Salmonella suppresses CXCR4 protein expression and tumor cell migration ability in B16F10 melanoma and LL2 lung carcinoma cells. Salmonella reduced CXCR4 protein expression through downregulating Protein Kinase-B (Akt)/Mammalian Target of Rapamycin (mTOR) signaling pathway. In cells transfected with constitutively active Akt plasmids, a reverse effect of Salmonella-induced inhibition of CXCR4 was observed. Tumor cells have chemotactic response to $\mathrm{CXCL12}$ in migration assay, and we found that Salmonella reduced tumor chemotactic response after CXCL1 2 treatment. The C57BL/6 mice were intravenously injected with B16F10 and LL2 cells pre-incubated with or without Salmonella, the tumor size and lung weight of Salmonella group had obviously decreased, indicating anti-metastatic effect that confirmed the findings from the in vitro experiments.
\end{abstract}

Key words: Salmonella, Metastasis, Migration, CXCR4, CXCL12

\section{Introduction}

The C-X-C chemokine receptor type 4 (CXCR4)/ stromal cell-derived factor 1 (SDF-1) axis has been reported to participate in the proliferation, migration, and invasion of several cancer types [1]. Metastatic spread of most cancers, including melanoma, lung cancers, generally leads to high mortality rate despite available interventions [2]. The CXCR4 in tumors correlates with tumor size, metastasis, and prognosis [3]. CXCR4 phenotype knockout strategies have been widely used to inhibit tumor metastasis mediated by the CXCR4/SDF-1 axis [3]. CXCR4 expression was reported to be mediated by several signaling mechanisms including protein kinase B (Akt) signaling that is known to modulate behavioral phenotypes in tumor such as anti-apoptosis, drug resistance and metastasis [4]. Thus, activation of the SDF-1/CXCR4/AKT pathway is important for tumor metastases and progression [5].

Salmonella, the Gram-negative, facultative anaerobic bacteria, has being used in cancer therapy [6]. Salmonella was used as tumor targeting therapeutic agents, because Salmonella was able to target small metastatic and primary tumors [7]. Previously, we showed that Salmonella can colonize 
small tumor nodules and enhance the survival of lung metastatic tumor mice [8]. In mouse metastatic tumor model, Salmonella can reduce the expression of Matrix metallopeptidase 9 (MMP-9) in tumors [9]. Herein, we identified Salmonella as a key factor in decreasing the migration of tumor cells. We also explored a therapeutic strategy to decrease the expression of CXCR4.

\section{Materials and Methods}

\section{Bacteria, Reagent, Cells, Plasmids, and Animals}

A vaccine strain of Salmonella choleraesuis [S. choleraesuis subsp. choleraesuis (Smith) Weldin serovar Dublin (ATCC 15480)] was obtained from Bioresources Collection and Research Center (Hsinchu, Taiwan) [10]. The resveratrol and 4',6-Diamidino-2-Phenylindole (DAPI) and mouse CXCL12/SDF-1 protein were purchased from Sigma-Aldrich (Sigma Aldrich, St. Louis, MO, USA). AMD3100 was purchased from Selleckchem (Sekkeckchem, Houston, TX, USA). Murine melanoma cells (B16F10) and murine lung cancer cells (LL2) were maintained in the Dulbecco's Modified Eagle's Medium (DMEM) containing 10\% fetal bovine serum gentamicin $(50 \mu \mathrm{g} / \mathrm{mL})$. A constitutively active AKT plasmid was a kind gift from Dr. C-Y Tsai. (Department of Molecular Immunology, Osaka University) [11]. The C57BL/6 mice were obtained from the National Laboratory Animal Center of Taiwan provided. The Laboratory Animal Care and Use Committee of the National Sun Yat-senUniversity approved the experimental protocol (permit number: 10829).

\section{Western Blotting and Transfection}

The protein content was used a bicinchoninic acid (BCA) protein assay (Pierce Biotechnology, Rockford, IL, USA) to determine the protein concentration. The sodium dodecyl sulfate polyacrylamide gel electrophoresis (SDS-PAGE) was used to fractionate protein samples. The protein samples were transfer to the hybond-enhanced chemiluminescence nitrocellulose membranes (Pall Life Science, Glen Cove, NY, USA). The membranes were incubated with various antibodies, including CXCR4 (GeneTex Inc. Irvine, CA, USA), the protein kinase B (AKT) (Santa Cruz Biotechnology, Santa Cruz, CA, USA), phosphorylation-AKT (Santa Cruz Biotechnology), mammalian targets of rapamycin (mTOR) (Cell Signaling, Danvers, MA, USA), phosphorylation-mTOR (Cell Signaling), p70 ribosomal S6 kinase (p-p70S6K) (Cell Signaling), phosphorylation-p70S6K (Cell Signaling), and $\beta$-actin
(Sigma-Aldrich). The appropriate horseradishperoxidase-conjugated secondary antibodies were used and enhanced chemiluminescence system (T-Pro Biotechnology, New Taipei City, Taiwan) to detect the protein-antibody complexes. Lipofectamine 2000 (ThermoFisher Scientific, Waltham, MA, USA) was used to transfect with the constitutively active AKT plasmids to cells.

\section{Wound-healing and Transwell Assays}

The wound-healing according to the manufacturer's instructions (IBIDI, Martinsried, Germany). The moving distance was detected after 24 $\mathrm{h}$ by using a microscope. The migration distances of untreated cells were set to $100 \%$ and were compared with cells treated with Salmonella. The cell migration according to the manufacturer's instructions (Transwell cultures (ThermoFisher Scientific). Cells were stained with DAPI and counted under a fluorescence microscope [9].

\section{Mouse Experiments}

The B16F10 $\left(10^{5}\right)$ and LL2 cells $\left(10^{5}\right)$ mixed with or without Salmonella (Multiplicity of infection, $\mathrm{MOI}=$ 100) for $1.5 \mathrm{~h}$ and C57BL/6 mice were injected with Salmonella-treated or non-treated-cells via the tail vein on Day 0. Tumor-bearing mice were sacrificed, and the lungs were removed, and weighed on day 18 .

\section{Statistical analysis}

Statistical analyses were performed by one-way ANOVA and Sidak's multiple comparison test relative to control groups using Prism 9. Any P value less than 0.05 is regarded statistically significant.

\section{Results}

\section{Salmonella reduced the migration of tumor cell}

The B16F10 mouse melanoma cells and LL2 mouse lung tumor cells were treated with Salmonella to examine the migration of tumor cells. The results of the wound-healing assay showed that the movement of tumor cells (B16F10 and LL2) was significantly inhibited compared with that of the PBS group after Salmonella treatment (Fig. 1A). The reduction of the motility of Salmonella-treated tumor cells was observed in Fig. 1A. Although Salmonella did not affect cell proliferation after a short period of infection, we further used Transwell assay to measure the migration of B16F10 and LL2 cells (Fig. 1B). As Fig 1B shown, Salmonella significantly reduced the movement of both types of tumor cells. Multiple tumor cell rely on CXCR4 and its ligand, SDF-1/CXCL12, to metastasis [12]. Meanwhile, CXCR4 inhibitor (AMD3100) suppressed tumor cell migration as Salmonella (Fig. S1). It is well known that 
high concentration of SDF1 suppressed chemotaxis. As Fig. S2 and Fig. 2 shown, Salmonella inhibited SDF-1-induced tumor cell migration by Transwell assay. Our data showed that Salmonella reduces the motility of tumor cells.

\section{Salmonella dose-dependently inhibited CXCR4 expression in tumor cells}

In this study, we found that Salmonella can inhibit SDF-1-induced tumor cell migration (Fig. 2). Furthermore, whether Salmonella can reduce tumor cell metastasis by inhibiting the receptor of SDF-1 (CXCR4), the protein expression in Salmonella-treated tumor cells were measured. We used Western blotting to evaluate the potential signaling pathways through which Salmonella has its anti-migration effects and examine the expression of CXCR4 and related signaling pathways (Fig. 3). The AKT/mTOR signaling is required for SDF-1 mediated migration in gastric carcinomas [13]. It is surprising that we found that Salmonella reduced the expression of CXCR4 in a dose-dependent manner in B16F10 and LL2 cells. Indeed, the expressions of phosphorylation AKT, phosphorylation mTOR, and phosphorylation p70S6K were reduced after Salmonella treatment in a dose dependent-manner (Fig. 3). We observed the correlation between CXCR4 and the AKT/mTOR/ p70S6K signaling pathway. These results suggested that Salmonella might inhibit CXCR4 expression through reducing the activity of AKT/mTOR/p70S6K signaling pathway.

\section{AKT/mTOR/p70S6K signaling pathway was involved in Salmonella-mediated CXCR4 expression}

In this study, Salmonella reduced the phosphorylation AKT and the expression of CXCR4. Dependent on our previous studies, the constitutively active AKT plasmids can be used to distinguish the correlation between targeting protein and the AKT/mTOR signaling pathway [6]. The AKT/ mTOR/p70S6K signaling pathway was reversed after transfecting constitutively active AKT plasmids (Fig. 4 $\mathrm{A}$ and B). The CXCR4 expression was reversed after being treated with Salmonella and constitutively AKT in B16F10 and LL2 cells, indicating that the Salmonellamediated decrease of CXCR4 through AKT/mTOR/ p70S6K signaling pathway (Fig. 4A and B). We used Transwell assay to evaluate the role of AKT in SDF-1induced tumor cell migration. As Fig. 4C shown, Salmonella significantly reduced the movement of SDF-1-treated B16F10 after transfecting constitutively active AKT plasmids. The results demonstrate that downregulation of phosphorylation $\mathrm{AKT} / \mathrm{mTOR}$ is required for Salmonella-mediated CXCR4 expression in tumor cells.
A

B16F10
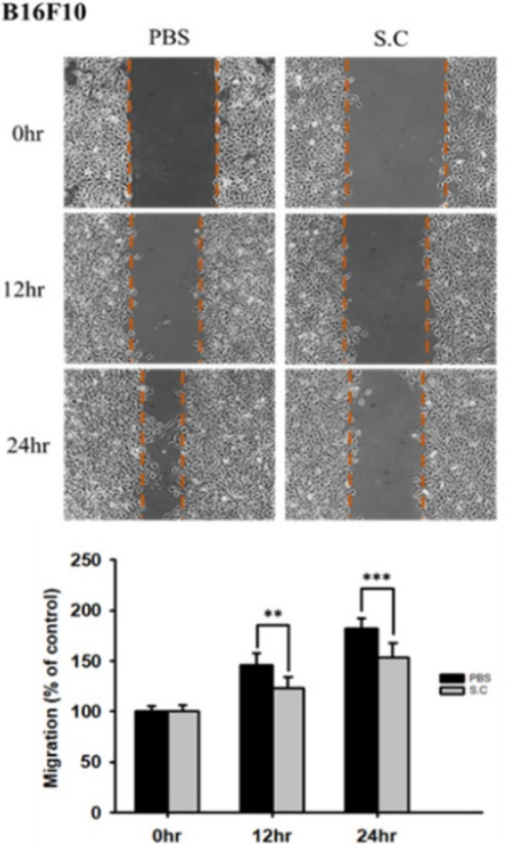

LL2
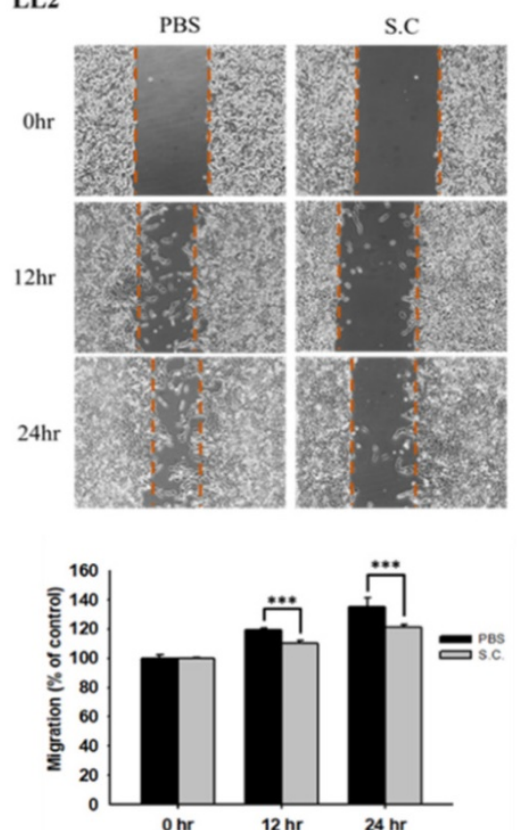

B

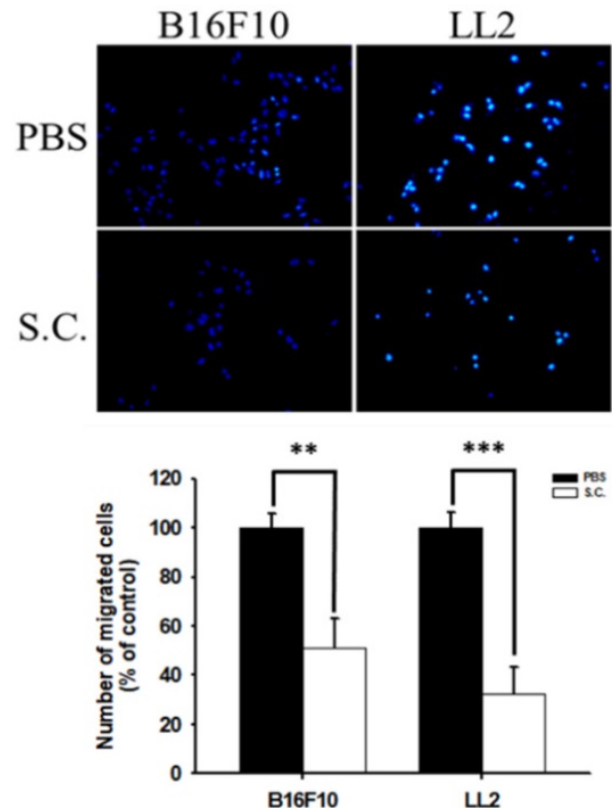

Figure 1. The cellular motility of B16F10 and LL2 cells after Salmonella (S.C.) treatment. The cells were co-cultured with Salmonella (MOI = 100) for 1.5 h. The motility distances of different groups of (A) B16F10 and LL2 cells were measured at different time points. (B) The B16F10 cells and LL2 cells were placed on the upper layer of Tranwell and then infected with Salmonella $(\mathrm{MOI}=100)$ for $90 \mathrm{~min}$. After $24 \mathrm{~h}$, the bottom layer of cells were stained with 4',6-diamidino-2-phenylindole (DAPI) and counted under a fluorescence microscope $(n=6$, mean $\pm S D$. *** $p<0.01$; *** $p<0.001)$. These replicates were used different passage of cells. 
B16F10
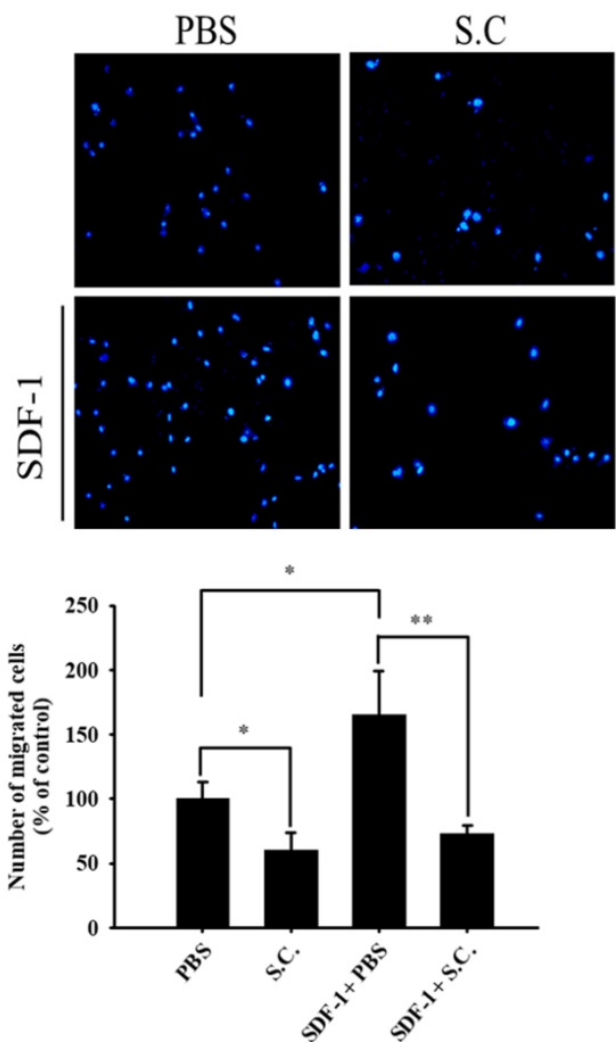

LL2
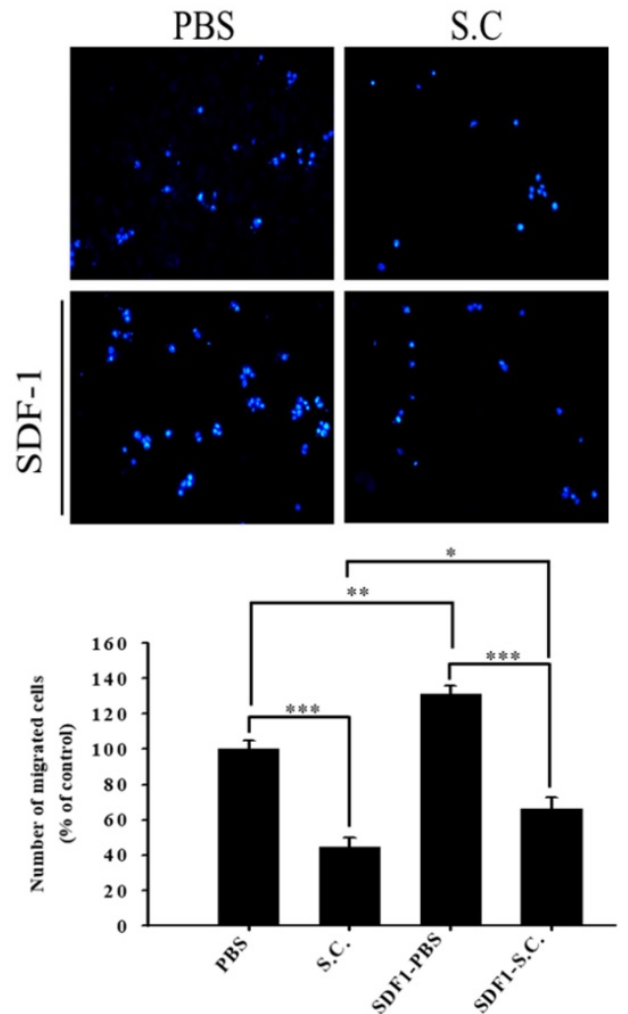

Figure 2. Tumor cells infected with Salmonella (MOI=100) were added to the upper chamber (with permeable membrane) in the $24-$ well plates and CXCL12 (100 ng/ml) were added in the lower chamber, then cultured for 24 hours. The cells which migrated through the permeable membrane were stained with DAPI and measured by fluorescensce microscope $(200 X)\left(n=6\right.$, mean \pm SD. $\left.{ }^{*} p<0.05 ; * * p<0.01 ; * * *<0.001\right)$. These replicates were used different passage of cells.

B16F10

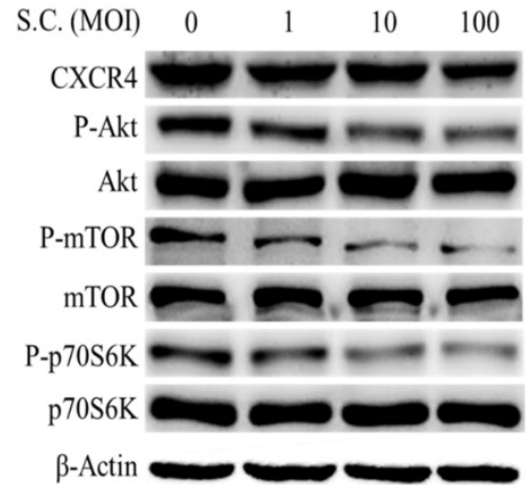

LL2

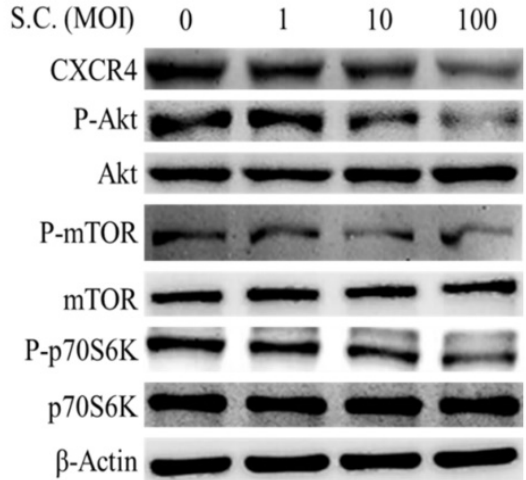

Figure 3. The CXCR4 expression in Salmonella-treated-B16F10 and-LL2 cells. The cells were co-cultured with Salmonella (MOI $=0-100)$ for $1.5 \mathrm{~h}$. The protein expression in B16F10 and LL2 cells was measured. The immunoblotting assay was repeated three times with similar results. Inserted values indicated relative proteins expression in comparison with $\beta$-actin.

\section{Salmonella reduced lung tumor nodules}

Moreover, we took advantage of a platform that screens for anti-metastatic molecules [14]. The tumor cells admixed with Salmonella $(\mathrm{MOI}=100)$ for $90 \mathrm{~min}$ and then intravenously injected into mice. Mice bearing metastatic nodules were sacrificed after inoculation of the tumors on day 18 day. The numerous pulmonary nodules were observed in the lungs from PBS-treated mice. However, the smaller and fewer tumor nodules were observed in the lungs from the mice treated with Salmonella (Fig. 5A). The weight of wet lung was measured to quantitatively determine tumor burden. The mice injected with B16F10 and LL2 tumor cells admixed with Salmonella had $36 \%$ and $22 \%$ lower wet lung weight, respectively, compared with those injected with cells admixed with PBS (Fig. 5B). Histological examination confirmed the macroscopic findings (Fig. 5C). Taken together, these results suggest that Salmonella affects tumor metastasis. 


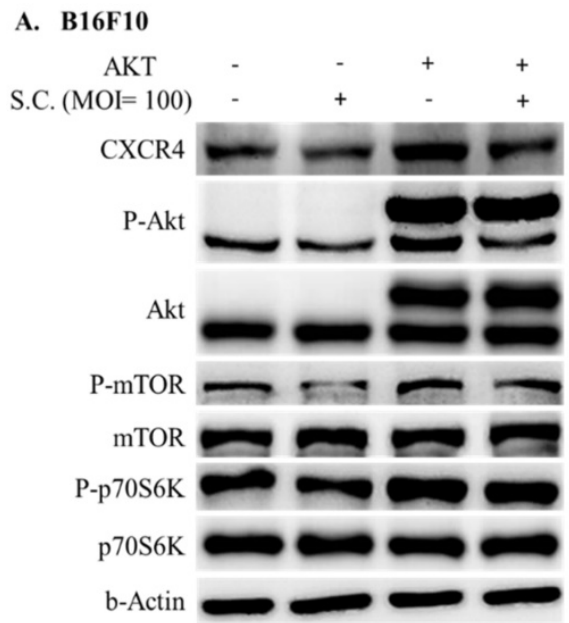

B. LL2

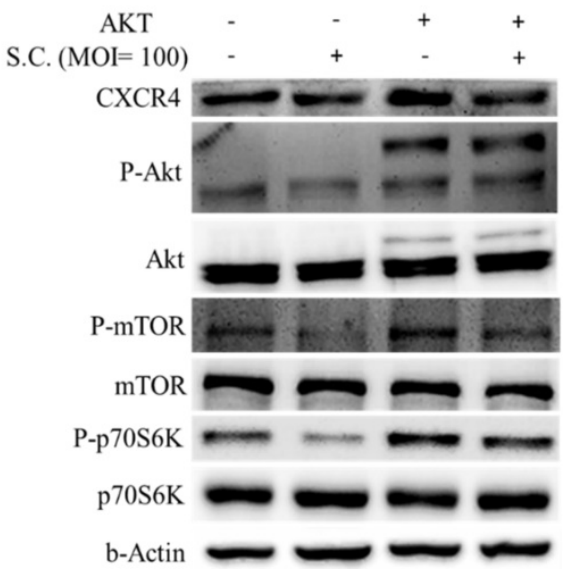

\section{C}

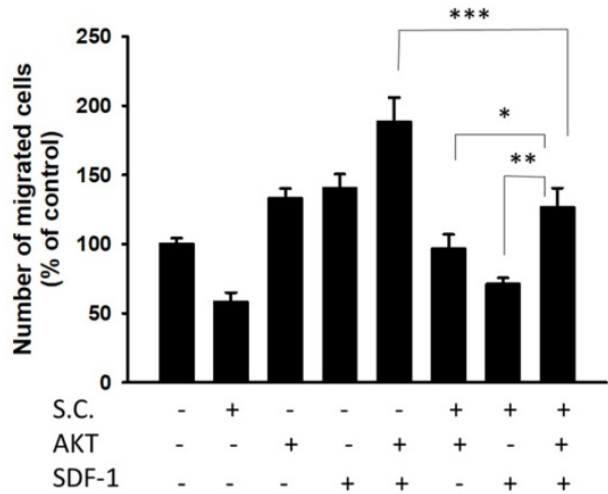

Figure 4. AKT signaling pathways were participated in Salmonella (S.C.)-mediated CXCR4 expression. The BI6F10 and LL2 cells were transfected with an active AKT plasmid. The cells were treated with Salmonella $(\mathrm{MOI}=100$ ) for $1.5 \mathrm{~h}$. The various protein expressions in B16F10 and LL2 cells was measured. The immunoblotting assay was repeated three times with similar results. Inserted values indicated relative proteins expression in comparison with $\beta$-actin. B16F10 cells infected with Salmonella (MOI=100) were added to the upper chamber (with permeable membrane) in the 24-well plates and CXCL12 $(100 \mathrm{ng} / \mathrm{ml}$ ) were added in the lower chamber, then cultured for 24 hours. The B16F10 cells were transfected with an active AKT plasmid. The cells which migrated through the permeable membrane were stained with DAPI and measured by fluorescensce microscope (200X). $\left(\mathrm{n}=6\right.$, mean \pm SD. $\left.{ }^{*} \mathrm{p}<0.05 ; * * \mathrm{p}<0.01 ; * * * \mathrm{p}<0.001\right)$. These replicates were used different passage of cells.

A

PBS

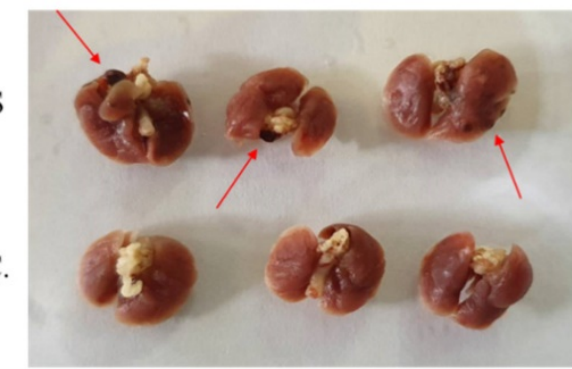

LL2

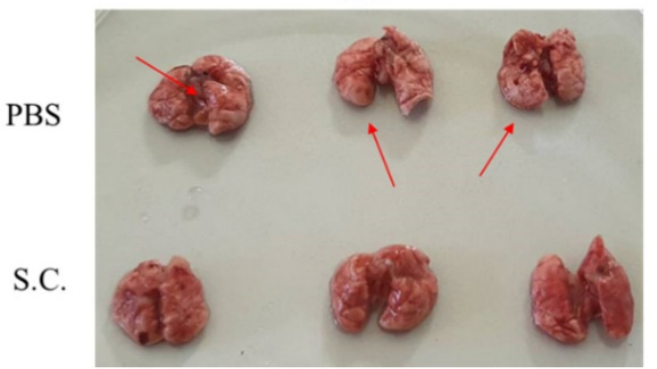

B

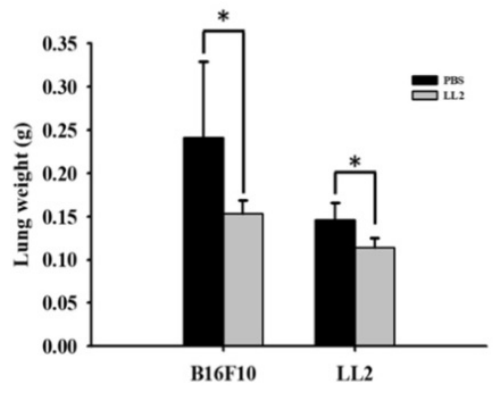

$\mathrm{C}$

$$
\text { B16F10 LL2 }
$$

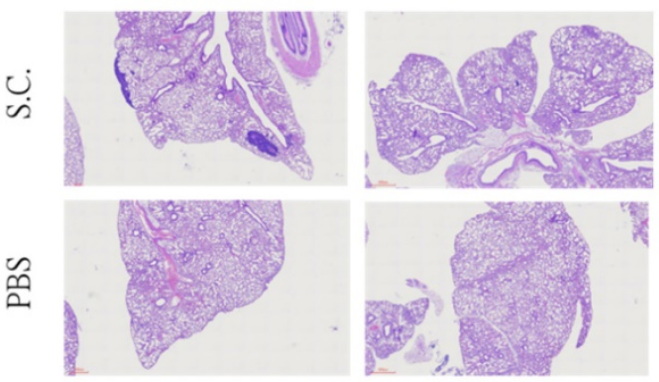

Figure 5. Salmonella reduced lung tumor nodules. The tumor cells admixed with Salmonella $(\mathrm{MOI}=100)$ for 90 min and then intravenously injected into mice. Mice bearing metastatic nodules were sacrificed after inoculation of the tumors on day 18 day. (A) Representative examples of metastatic pulmonary nodules produced 18 days after intravenous injection of tumor cells. (B) The anti-tumor effect of Salmonella was measured by lung weight. $(\mathrm{n}=3$, data are expressed as mean \pm SD. * $\mathrm{P}<0.05)(\mathbf{C})$ Histological examination of pulmonary tumor nodules at day 18 after tumor inoculation (40X). 


\section{Discussion}

It is surprising that Salmonella not only inhibits primary tumor growth but also prevent metastasis. This suggests that Salmonella, which inhibits primarily AKT/mTOR signaling pathway, may represent promising a target agent preventing metastasis of many aggressive tumors that use CXCR4 for the guided migration of tumor cells from primary tumors to secondary colonization sites. The SDF-1 is often produced at high levels by metastatic target organs including lung, liver and bone [15]. CXCR4 upregulation in tumor cells enhanced the migratory potential of tumor cells towards SDF-1 producing organs [15]. The CXCR4-inhibitors a promising class of anti-tumor agents that should be further explored in clinical trials. CXCR4 inhibitors are in clinical use as bone marrow stem cell or progenitor cell mobilizers [16].

Therefore, the ability of Salmonella to target multiple tumors from distant sites makes it an ideal anti-tumor agent over some other cancer therapeutic agents limited to local administration. The anti-CXCR4 activity was specific in tumor sites by tumor-targeting Salmonella. The special effects of Salmonella can inhibit primary tumor growth and reduce metastasis from primary tumor. Previously, Salmonella had that activity that is anti-angiogenesis by reducing hypoxia-inducible factor 1-a expression [11]. Interestingly, Salmonella infected with porcine and analyzed RNA profiles from the mesenteric lymph nodes [17]. CXCR4 mRNA was upregulated in this model. Salmonella may enhance immune cell response, but reduced the epithelial-mesenchymal transition (EMT) of tumor. Salmonella can break tumor immune tolerance and reduce tumor metastasis.

In a tumor microenvironment, two main producers of Interferon- $\gamma$ (IFN- $\gamma)$ are natural killer (NK) cells, and tumor-specific cytotoxic CD8+ T lymphocytes (CTLs). Th1 polarized CD4+ $\mathrm{T}$ helper cells also secrete IFN- $\gamma$ that help in the promotion and maintenance of anti-tumor CTL responses. Previously, we showed that Salmonella significantly upregulated IFN- $\gamma$ which may be responsible for recruiting peripheral immune cells to the tumor in wild-type mice, but not in T-cell-deficient mice [18]. We suggested the $\mathrm{T}$ cell is involved in the regulation of Salmonella-induced host antitumor immunity in tumor-bearing mice. Thus, our studies may provide a cellular basis for understanding the recruitment of effector immune cells and the synergism between the oncolytic effect of Salmonella and adaptive antitumor immune mechanisms. Salmonella in tumor induced signal transduction by multiple mechanisms including competition for nutrients, stimulation of immune response [19]. Moreover, toxin from Salmonella may induce the apoptosis of tumor. As Salmonella replication in tumors and subsequent lysis of tumor cells may induce cell-mediated immune responses to tumor cells, higher oncolysis could account, in part, for an increased infiltrate of immune cells in tumors. The cells undergoing bacteria-induced cell death exhibit heterogeneous morphological features.

Taken together, our study suggests that Salmonella is sufficient to inhibit primary tumor growth and CXCR4-dependent migration and metastasis in vivo.

\section{Supplementary Material}

Supplementary figures.

http://www.medsci.org/v18p2835s1.pdf

\section{Acknowledgements}

This work was supported by Ministry of Science and Technology, Taiwan (MOST 109-2326-B-110001-MY3), Kaohsiung Veterans General Hospital/ National Sun Yat-sen University research project (KSVNSU110-013), Kaohsiung Municipal Ta-Tung Hospital research project, and Higher Education Sprout Project.

\section{Author Contributions}

T.H.L., G.Y.L. and C.H.L. conceived, designed and revised the experiments; T.H.L. M. H. Y., Y.C.Y. and G.Y.L. performed the experiments; G.Y.L. and C.H.L analyzed the data; C.H.L contributed reagents/ materials/analysis tools; T.H.L. and C.H.L wrote the paper.

\section{Competing Interests}

The authors have declared that no competing interest exists.

\section{References}

1. Chen L, Chen $\mathrm{Q}, \mathrm{Wu}$ Y, Zhu M, Hu J, Zhuang Z. MTSS1 inhibits colorectal cancer metastasis by regulating the CXCR4/CXCL12 signaling axis. Int J Mol Med. 2021; 47(5):65.

2. Mei L, Liu Y, Zhang Q, Gao H, Zhang Z, He Q. Enhanced antitumor and anti-metastasis efficiency via combined treatment with CXCR4 antagonist and liposomal doxorubicin. J Control Release. 2014; 196:324-331.

3. Ieranò C, D'Alterio C, Giarra S, Napolitano M, Rea G, Portella L, Santagata A, Trotta AM, Barbieri A, Campani V, Luciano A, Arra C, Anniciello AM, Botti G, Mayol L, De Rosa G, Pacelli R, Scala S. CXCL12 loaded-dermal filler captures CXCR4 expressing melanoma circulating tumor cells. Cell Death Dis. 2019; 10(8):562.

4. Wang DD, Jiang MY, Wang W, Zhou WJ, Zhang YW, Yang M, Chen JY, Wei W. Paeoniflorin-6'-O-benzene sulfonate down-regulates CXCR4Gbetagamma-PI3K/AKT mediated migration in fibroblast-like synoviocytes of rheumatoid arthritis by inhibiting GRK2 translocation. Biochem Biophys Res Commun. 2020; 526(3):805-812.

5. Dillenburg-Pilla $\mathrm{P}$, Patel $\mathrm{V}$, Mikelis $\mathrm{CM}$, Zárate-Bladés $\mathrm{CR}$, Doçi $\mathrm{CL}$, Amornphimoltham P, Wang Z, Martin D, Leelahavanichkul K, Dorsam RT, Masedunskas A, Weigert R, Molinolo AA, Gutkind JS. SDF-1/CXCL12 induces directional cell migration and spontaneous metastasis via a CXCR4/Gai/mTORC1 axis. FASEB J. 2015; 29(3):1056-1068. 
6. Chen $\mathrm{MC}$, Pangilinan $\mathrm{CR}$, Lee $\mathrm{CH}$. Salmonella Breaks Tumor Immune Tolerance by Downregulating Tumor Programmed Death-Ligand 1 Expression. Cancers (Basel). 2019; 12(1):57.

7. Wang WK, Chiang WC, Lai CH, Lee CH. Salmonella-Mediated Cytolethal Distending Toxin Transfer Inhibits Tumor Growth. Hum Gene Ther. 2018; 29(11):1327-1335.

8. Lee $\mathrm{CH}, \mathrm{Wu} \mathrm{CL}$, Tai YS, Shiau AL. Systemic administration of attenuated Salmonella choleraesuis in combination with cisplatin for cancer therapy. Mol Ther. 2005; 11(5):707-716.

9. Tsao YT, Kuo CY, Cheng SP, Lee CH. Downregulations of AKT/mTOR Signaling Pathway for Salmonella-Mediated Suppression of Matrix Metalloproteinases-9 Expression in Mouse Tumor Models. Int J Mol Sci. 2018; 19(6):1630.

10. Yang CJ, Chang WW, Lin ST, Chen MC, Lee CH. Salmonella Overcomes Drug Resistance in Tumor through P-glycoprotein Downregulation. Int J Med Sci. 2018; 15(6):574-579.

11. Tu DG, Chang WW, Lin ST, Kuo CY, Tsao YT, Lee CH. Salmonella inhibits tumor angiogenesis by downregulation of vascular endothelial growth factor. Oncotarget. 2016; 7(25):37513-37523

12. Saxena R, Wang Y, Mier JW. CXCR4 inhibition modulates the tumor microenvironment and retards the growth of B16-OVA melanoma and Renca tumors. Melanoma Res. 2020; 30(1):14-25.

13. Chen G, Chen SM, Wang X, Ding XF, Ding J, Meng LH. Inhibition of chemokine (CXC motif) ligand 12/chemokine (CXC motif) receptor 4 axis (CXCL12/CXCR4)-mediated cell migration by targeting mammalian target of rapamycin (mTOR) pathway in human gastric carcinoma cells. J Biol Chem. 2012; 287(15):12132-12141.

14. Chen MC, Chang WW, Kuan YD, Lin ST, Hsu HC, Lee CH. Resveratrol inhibits LPS-induced epithelial-mesenchymal transition in mouse melanoma model. Innate Immun.2012; 18:685-693.

15. Müller A, Homey B, Soto $\mathrm{H}$, Ge N, Catron D, Buchanan ME, McClanahan $\mathrm{T}$, Murphy E, Yuan W, Wagner SN, Barrera JL, Mohar A, Verástegui E, Zlotnik A. Involvement of chemokine receptors in breast cancer metastasis. Nature. 2001; 410(6824):50-56

16. Walenkamp AME, Lapa C, Herrmann K, Wester HJ. CXCR4 Ligands: The Next Big Hit? J Nucl Med. 2017; 58(Suppl 2):77S-82S.

17. Uthe JJ, Stabel TJ, Zhao SH, Tuggle CK, Bearson SM. Analysis of porcine differential gene expression following challenge with Salmonella enterica serovar Choleraesuis using suppression subtractive hybridization. Vet Microbiol. 2006; 114(1-2):60-71.

18. Lee $\mathrm{CH}, \mathrm{Hsieh} \mathrm{JL}, \mathrm{Wu} \mathrm{CL}, \mathrm{Hsu}$ PY, Shiau AL. T cell augments the antitumor activity of tumor-targeting Salmonella. Appl Microbiol Biotechnol. 2011; 90:1381-1388.

19. Pangilinan $\mathrm{CR}$, Lee $\mathrm{CH}$. Salmonella-based targeted cancer therapy: updates on a promising and innovative tumor immunotherapeutic strategy. Biomedicines. 1029; 7 pii: E36. 